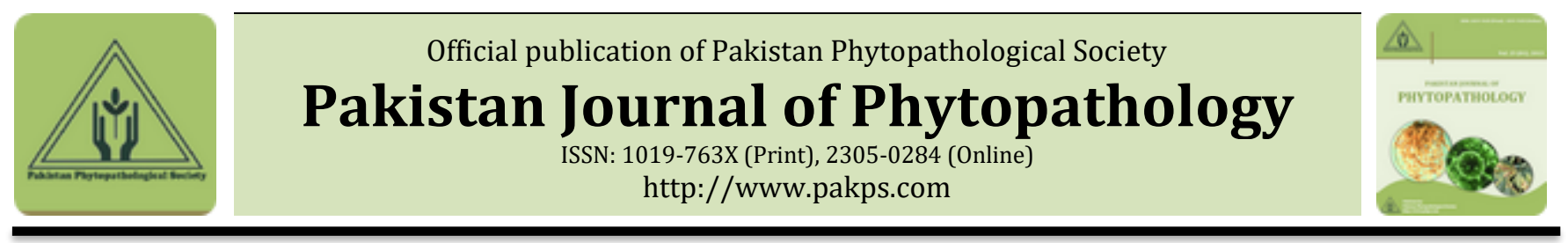

\title{
BIOLOGICAL CONVERSION OF POTATO SPINDLE TUBER VIROID (PSTVD) RNA INTO NONINFECTIOUS ENTITY BY A SINGLE BASE SUBSTITUTION IN NICOTIANA BENTHAMIANA VAR DOMIN
}

\author{
Zaryab K. Sial, Farah Khan* \\ Department of Botany, Lahore College Women University, Lahore, Pakistan.
}

A B S T R A C T

\begin{abstract}
It is matter of common understanding that when we inoculate some strain of pathogen to model plant, it moves from source to sink. The Potato spindle tuber viroid (PSTVd) was first identified viroid of family Pospiviroidae, was isolated from Potato leaves i.e. natural host and submitted to Genebank (Accession \# MK303578). The PSTVd is $a$ single stranded circular RNA having 359 nucleotides which causes mild to severe symptoms in experimental host. Nicotiana benthamiana was selected as model targeted host and inoculated with mutated PSTVd (at base position 171). A single base change created at base position 171 of loop motif ' 26 ' from A to U (T) has stopped PSTVd replication and make it noninfectious The RNA was extracted from host and converted into cDNA and sequenced again. The inoculated plant with a single base change showed no infection. No infection symptoms on experimental host plant.. The results of sequencing and BLAST and Mfold (version 3.0) confirmed a single base change at position 171 of Loop 26 of PSTVd. This was a unique approach to change genetic sequence of PSTVd to minimize/stop its infection and spread.
\end{abstract}

Keywords: Potato spindle tuber viroid, beta marceptaethanol, RT-PCR.

\section{INTRODUCTION}

Potato Spindle Tuber Viroid (PSTVd) is first recognized viroid belongs to family Pospiviroidae [which are considered as the subviral RNA plant pathogens in having single stranded circular RNAs, ranges from 260-450 nucleotides base pairs which cause infection to host (Bagherian et al., 2014). The reproductive mechanism of viruses and viroids is same. It takes the control of the reproductive factory of host plant for the synthesis of its own RNA. It is also considered that these infectious RNAs as an original ancestor of small viral RNAs, Which have no ability to code some amino acids for protein synthesis and have to rely on hosts for its replication. These qualities differentiate them from satellite RNAs and plant viruses (Boonham et al., 2004).

The movements of these RNA pathogens like PSTVd is very

\begin{tabular}{l} 
Submitted: October 17, 2018 \\
Revised: December 27, 2018 \\
Accepted for Publication: May 14, 2019 \\
* Corresponding Author: \\
Email: drfarah_khann@yahoo.com \\
(c) 2017 Pak. J. Phytopathol. All rights reserved. \\
\hline
\end{tabular}

important for understanding plant physiology and development (Bostan et al., 2004). The interaction of plant host and pathogen is also very important for establishing a systemic infection by viroids. Increasing evidences have proved that these kinds of processes are regulated by specific type of RNAs (Bostan et al., 2004).

The PSTVd provides a unique model to understand function and structure of RNA motifs for causing an infection. The different Loops of PSTVd have been worked out before for understanding their functions. A single base substitution from $C$ to $G, A$ to $U(T)$ and reversion can change pathogenic efficiency of PSTVd was first time reported by Wassenegger (Candresse et al, 2010). During the last decades, several loops motifs of PSTVd have been investigated for this purpose (Constable and Moran 1996; Di Serio, 2007; Ding, 2010).

The concept of site directed mutagenesis in the PSTVd has opened new era of discussion. The inoculation of PSTVd on leaves of host plant cause possible movement from source to sink. A single base substitution can produces a new mutant which don't trafficks into host plant (Ding and Itaya 2007; Fels et al., 2001). So we have focused on loop 26 at 
base position 171 analysis and single change was made at position $171(\mathrm{~A}-\mathrm{T})$ by site directed mutagenesis.

Nicotiana benthamiana is the model species for plant virology and contemplating plant-organism collaborations. It is additionally a model for certain plant physiological activity. We have tried to evaluate the mechanism of viroids pathogenicity using PSTVd mutant as an experimental system. In this concern we reported that a special change in at position 171 of viroidal genome can help to decrease its infection.

\section{MATERIAL AND METHODS}

Isolation of PSTVd RNA: The first and formost step of our study was to isolate PSTVd from infected plants i.e potato., which were recognized on the basis of symptoms as mentioned in literature (Di Serio, 2007). The PSTVd RNA was solated from infected Plants by modified Trizol method. The prior addition of beta marceptaethanol (BME) in trizol, helped to improve fine extraction of PSTVd RNA (1 $\mu \mathrm{l}$ BME/1ml Trizol) /The infected frozen plant tissues were ground in $500 \mu \mathrm{l}$ of modified Trizol reagent. For phase separation added $100 \mu \mathrm{l}$ of chloroform reagent and vortexed for 20 seconds then incubated at room temperature for almost 30 minutes. This sample was again centrifuged centrifuged at $13000 \mathrm{rpm}$ for 15 minutes. Two phases were formed i.e lower red interphase and colourless aqueous phase. The upper phases was collected in separated tubes then added $250 \mu \mathrm{l}$ of cold Isopropanol at room temperatureand left for 10 minutes. After incubation period of ten minutes this mixture was again centrifuged at $13000 \mathrm{rpm} 4{ }^{\circ} \mathrm{C}$ for 20 min. The pellet was collected and $500 \mu \mathrm{l} 75 \%$ ethanol was added and centrifuged again for three minutes at 750rpm ( $1 \mathrm{ml}$ of $75 \%$ ethanol was added as per $1 \mathrm{ml}$ of trizol reagent). The pellet was dried and suspended in $500 \mu \mathrm{l}$ of Trizol reagent. The storing pellate in Trizol for required period of time was another modification of our described method. RT-PCR was run with PSTVd specific primers (Gozmanova, 2003). The presence of PSTVd was confirmed by BLAST and Sequencing.

Site Directed Mutagenesis: The Loop 26 of PSTVd consisting of 8 nucleotides (ACAUUCCU). The first nucleotides was selected and Primer wwasdesigned (AAUCAGGGCCCUUCCUUU) for cDNA (AATCAGGGCCCTTCCTTT) with single base change from $A$ to $U$ (T) at position 171. The Reverse transcription process was used to make PSTVd mutant by the protocol (Hammond, 1992).

Plant growth and viroidal infections: The
N.benthamiana plants were grown in control chambers maintained at 16 hours light and 8 hours dark photoperiod. The inoculation of this mutant (250ng/leaf) was done on first two true leaves of host plant. Before inoculation small abrasion were also made on leaves with carborundum powder. The original wild type extracted RNA was also inoculated as positive control at the same time. The Diethyl Pyrocarbonate (DEPC) treated water was used for mock inoculation. Twenty one days of post inoculation the RNA was extracted from inoculated leaves (Postive \& Negative control) .

\section{RNA Extraction with Modified Trizol Protocol}

cDNA Synthesis: For completion of experiment extracted RNAs were converted in to cDNA on same day. The GScript First strand synthesis Kit (Cat No MB 3050050) was used to convert RNA into cDNA following manufacturer's instruction.. Extracted RNA $(2 \mu \mathrm{l})$ was added in eppendorf and Kit's component I .e Oligo (dT) 1 $\mu \mathrm{l}$ and dNTPs mix $(1 \mu \mathrm{l})$ were mixed in same tubes and centrifuged for $5 \mathrm{~min}$ at $13000 \mathrm{rpm}$. The $13 \mu \mathrm{l}$ of nuclease free water was added in centrifuged sample. It was heated in water bath for almost 3-5 min at $65^{\circ} \mathrm{C}$. when incubation period was completed the whole mixture was spun shortly on vortex and shifted on ice briefly. $5 \mathrm{x}$ ist strand buffer of kit was also added as $4 \mu \mathrm{l}$. Gscript Rtase and DTT (0.1M) were added $1 \mu \mathrm{l}$ Respectively (Ding, 2010).

Agarose GEL run: Agarose Gel was run and bands were noted and scored. The quality of extracted PSTVd cDNA was checked on a $1 \%$ agarose gels stained with Ethidium bromide and visualized under $\mu$ utraviolet light. The quantity of was PSTVd RNA and cDNA also noted with spectrophotometry. All samples were found between 1-2 $\mathrm{nm}$ UV-range. The quantification of cDNA was also performed with TMNanodrop 2000/2000c Thermo Fisher Scientific V1.0 The larger peaks at260/280nm were obtained for purified sample. The Visualization of bands were done in gel documentation system and was photographed. The high molecular and high quality bands were seen and scored for good quality c DNA (Hammond, 1992).

Sequencing: The PSTVd cDNA were submitted to BioBasic Canada inc for Sanger Dideoxy Sequencing. The obtained seq files (ID: Ist Base 3332384_3P) were analyzed Bioedit and FASTA sequence was submitted to gene bank and got accession number i.e Accession \# MK.303578. The RNA unfolding studies $\mathrm{M}$ fold also confirmed the base change at position 171 of viroidal genome. 


\section{RESULTS}

In order to have a look into function of loop 26, a single change was made at base position 171 of PSTVd (Figure 1). As mentioned earlier that at a single nucleotide change at any position of PSTVd genome can make it non infectious (Figure 2 and 3). The Original PSTVd strain and mutant was rubbed on first two leaves of Nicotiana benthamiana. The host plant leaves were plucked after 21 days of post inoculation (Table 1). The RNA from both plants was extracted and subjected RT-PCR. The cDNA obtained gave clear and sharp bands for PSTVd and cloudy bands for the mutants (Figure 4):

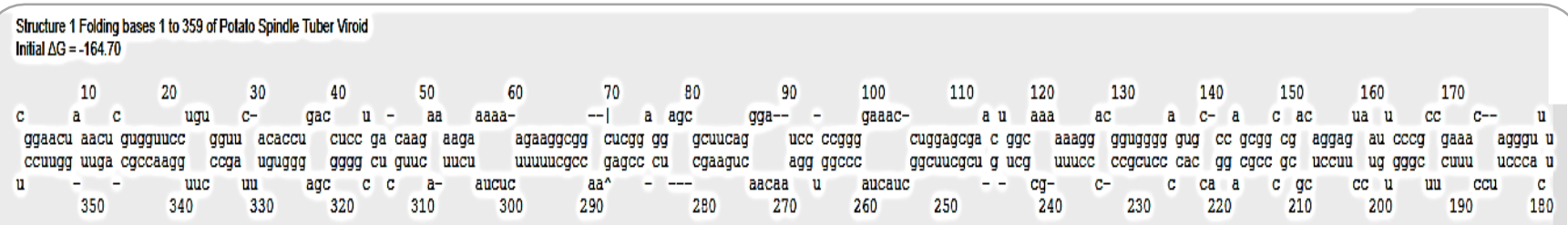

Figure 1. Genomic map of PSTVd with loops and motif having 359 nucleotides.

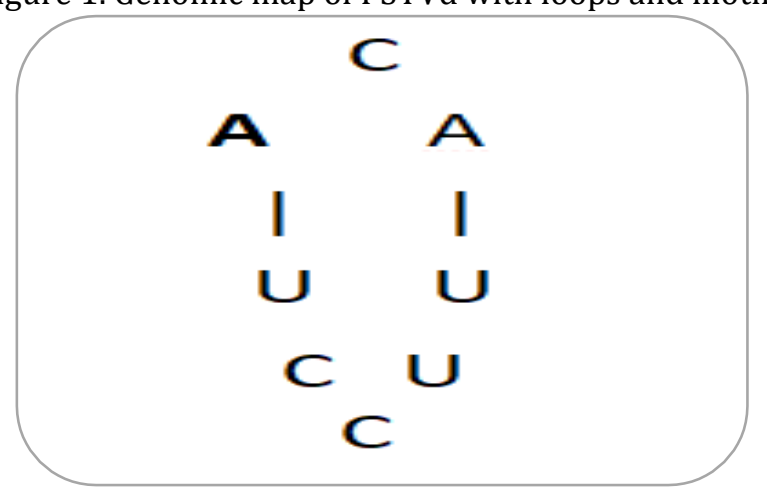

Figure 2. Loop 26 of PSTVd having sequence ACAUUCCUA

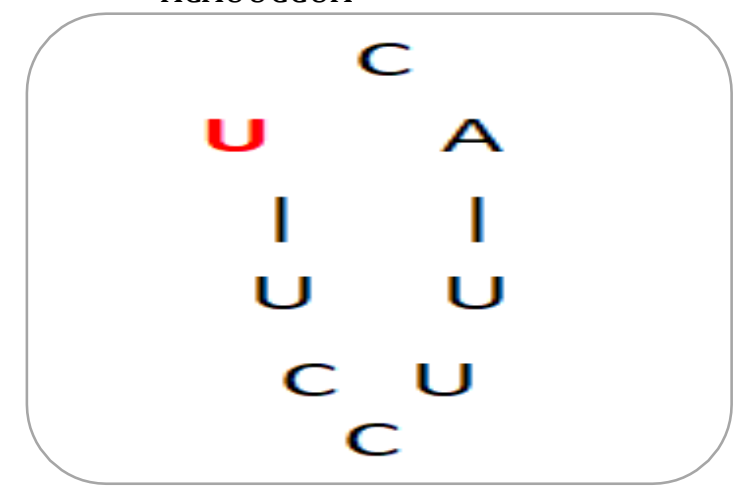

Figure 3. Loop 26 of PSTVd mutant UCAUUCCUA A U

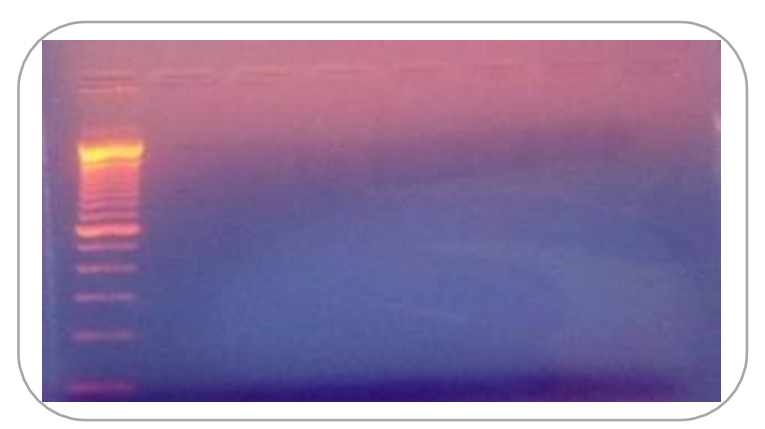

Figure 6. Absence of bands of amplified PSTVd mutant which show no infection symptoms.

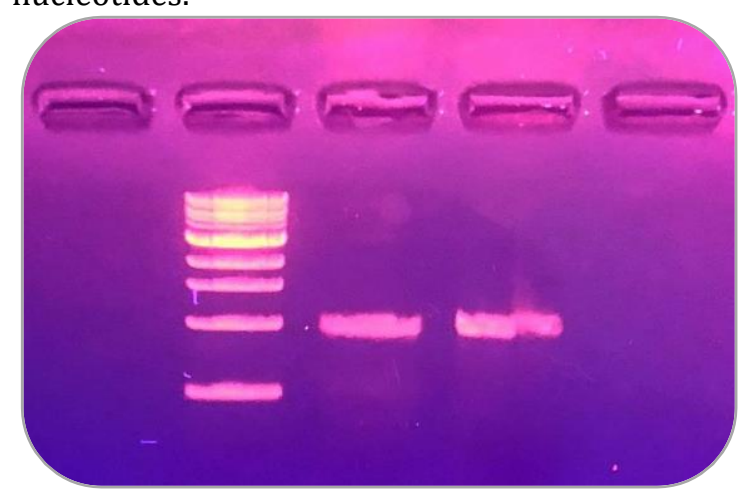

Figure 4. Sharp bands of PSTVd RNA extracted from PSTVd inoculated RNA

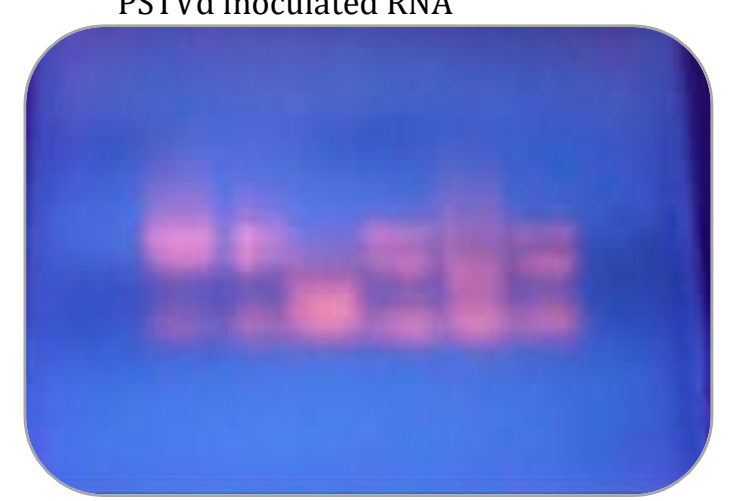

Figure 5. Light bands of amplified PSTVd mutants before inoculation to host Plants

Results have indicated that very sharps bands obtained with wild PSTVd strain. The generated mutant produced unclear merged band cloudy mutant (Figure 5). When it was inoculated onto Host Plant surface and RNA was extracted after three weeks. It showed no bands on gel (Figrue 6). It clearly indicated that transition from $A$ to $U$ (T) (in cDNA U is changed into T) make a trafficking incompetent mutant (Figure 7). Two to three PCRs were optimized to check presence/absence of PSTVd bands. This could not be detected into a inoculated leaves. 
Table 1. Morphological 1 changes observed in N. benthamiana infected by PSTVd mutant 21 days of post inoculation.

\begin{tabular}{lllllll}
\hline $\begin{array}{l}\text { Sr } \\
\text { No. }\end{array}$ & $\begin{array}{l}\text { Mutation } \\
\text { Sequance }\end{array}$ & $\begin{array}{c}\text { Number of plants } \\
\text { inoculated }\end{array}$ & $\begin{array}{l}\text { Disease } \\
\text { Symptoms }\end{array}$ & $\begin{array}{l}\text { Number of Plant } \\
\text { showed trafficking }\end{array}$ & $\begin{array}{l}\text { Trafficking } \\
\text { efficiency\% }\end{array}$ & $\begin{array}{l}\text { Pathogenic } \\
\text { efficiency }\end{array}$ \\
\hline 1 & $\mathrm{~A} \rightarrow \mathrm{U}$ & $40 \pm 0.52$ & $\begin{array}{l}\text { No obvious } \\
\text { symptoms }\end{array}$ & $0.0 \pm 0.33$ & $00 \pm 0.00$ & - \\
\cline { 2 - 6 } 2 & $40 \pm 0.52$ & $\begin{array}{l}\text { No obvious } \\
\text { symptoms }\end{array}$ & $1.0 \pm 0.33$ & $10 \pm 0.00$ & - \\
\cline { 2 - 6 } 3 & $40 \pm 0.52$ & $\begin{array}{l}\text { No obvious } \\
\text { symptoms }\end{array}$ & $0.0 \pm 0.33$ & $0.0 \pm 0.00$ & - \\
\hline
\end{tabular}

The mean with different letter in each column are significantly different according to Duncan's multiple range test $(0.005 p$ value $) \pm=$ Standard error

It was recorded in (Table 1) that trafficking/ transport of PSTVd completely changed after base change in viroidal genome.

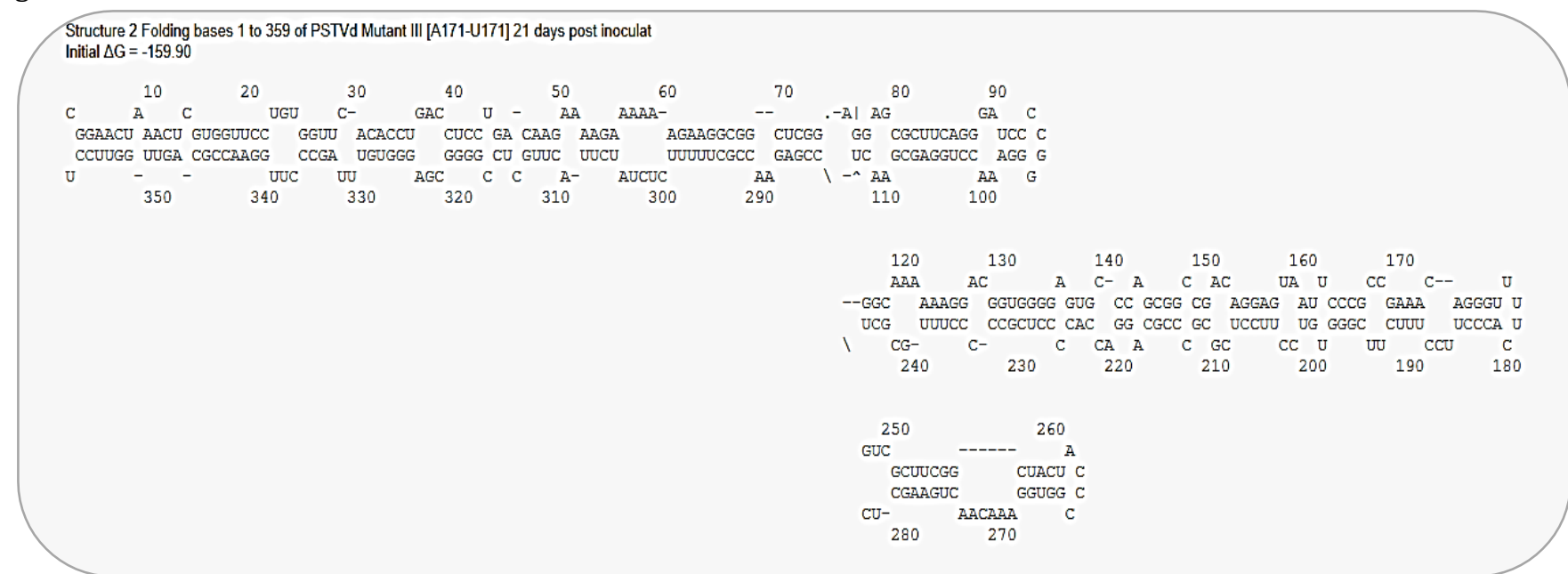

Figure 7. Fold analysis of PSTVd Mutant with Base Position number $171 \mathrm{~A}$ is replaced with U (A171-U171) after 21

\section{DISCUSSION} days of inoculation to N.benthamiana

The extraction of PSTVd and trafficking has been reported as an area of genomics from last decades by many researchers in different countries (Arif et al., 2005; Ding, 2010). The RNA of pathogens moves from cell to cell and cell to organ through special opening 'plasmodesmata' (Fels et al., 2001). Increasing evidences have reported that these process are regulated by RNA movements inside the cells (Gozmanova, 2003; Guner et al., 2012) The study of PSTVd RNA and its structural loops motifs has enabled us to investigate function-structure relationships for short and long distance transport. The unique feature of PSTVd make it a model to study different loops and motifs (Ding, 2010). About 11 to 14 loops have been PSTVd genomic RNAs have already been investigated in Nicotiana benthamiana (Hammond and Owens 1987). The nucleotides base substitution from A to U, U to G A to $\mathrm{T}$ has disturbed the function of loops which indirectly has effected movements of PSTVd in host plants (Kasai et al., 2013). The present research is different and first time reported in details for gain and loss of functions of PSTVd mutants. Sometime a base substitution A/C can lead to new Loop formation and function of PSTVd movements restore as happened in loop19 (Kasai et al., 2013). The inoculation of PSTVd RNA has produced the symptoms of reduced growth and colour change in leaves. These symptoms have already been reported by the work of Kolonko et al., 2006). The presence of viroids RNA was confirmed by sequencing of cloned products that have amplified in PCR with PSTVd specific Primers with no base changes (Kolonko et al., 2006). A total of 10 to 12 microgram of PSTVd and PSTVd mutant was used for inoculation onto Nicotiana benthamiana plants. Three weeks later the inoculated plants leaves were harvested. The total RNA was extracted and cDNA was prepared similarly discussed by the research of Ding (Ling et al., 2014). The Plants inoculated with PSTVd show symptoms but mutant inoculated plants have not shown any 
symptoms and gave no bands when run on a gel. This work was in accordance with the work of other researchers of same domains Mackie et al., 2015; Mahfouze, 2008). The site directed generated mutant of PSTVd have not shown any symptoms to model plant (Owens, 2007; Owens and Baumstark 2007) The sequence comparison of PSTVd FASTA with other sequence has shown a change at position 171 out of 359 nucleotides by sequencing analysis.

\section{CONCLUSION}

The presented study have proved as an outstanding and efficient method for site directed mutation of PSTVd RNA, its mutant generation and amplification from infected plants like Potatoes. The single base substitution of PSTVd and making it non infectious is a new addition to existing research.

Conflict of Interests Statement: Authors declare that there is no conflict of interest for publishing this study.

\section{ACKNOWLEDGEMENT}

The and Molecular genetics and Plant Biotechnology Lab in the Department of Botany, Lahore College for Women University, provided all the necessary items during present studies. Some part of the research was also executed under the Guidance of Sir M. Dr M. Ashfaq and help of hard working student Mubarak Ali Anjum in Institute of agriculture, University of Punjab. We are very thankful to kind cooperation of different students of both labs.

\section{REFERENCES}

Arif, M., A. Ahmad, M. Ibrahim and S. Hassan. 2005. Occurrence and distribution of virus and viruslike diseases of citrus in north-west frontier province of Pakistan. Pakistan Journal of Botany, 37: 407.

Bagherian, S., K. Izadpanah, H. Hamzehzarghani, M. Djavaheri and Y. Ghasemi. 2014. Effect of infection by mild variant of potato spindle tuber viroid on metabolic profiles of tomato cultivar'Rugers'. Iranian Journal of Plant Pathology, 50.

Boonham, N., L. G. Pérez, M. Mendez, E. L. Peralta, A. Blockley, K. Walsh, I. Barker and R. Mumford. 2004. Development of a real-time RT-PCR assay for the detection of Potato spindle tuber viroid. Journal of virological methods, 116: 139-146.

Bostan, H., X. Nie and R. P. Singh. 2004. An RT-PCR primer pair for the detection of Pospiviroid and its application in surveying ornamental plants for viroids. Journal of Virological Methods, 116: 189-
193.

Candresse, T., A. Marais, X. Tassus, P. Suhard, I. Renaudin, A. Leguay, F. Poliakoff and D. Blancard. 2010. First Report of Tomato chlorotic dwarf viroid in Tomato in France. Plant Disease, 94: 633633.

Constable, F. and J. Moran. 1996. PCR protocols for the detection of chrysanthemum stunt and potato spindle tuber viroids. Agriculture Victoria, Institute for Horticultural Development.

Di Serio, F. 2007. Identification and characterization of Potato spindle tuber viroid infecting Solanum jasminoides and S. rantonnetii in Italy. Journal of Plant Pathology: 297-300.

Ding, B. 2010. Viroids: Self-replicating, mobile, and fastevolving noncoding regulatory RNAs. Wiley Interdisciplinary Reviews: RNA, 1: 362-375.

Ding, B. and A. Itaya. 2007. Viroid: A Useful Model for Studying the Basic Principles of Infection and RNA Biology. Molecular Plant-Microbe Interactions, 20: 7-20.

Fels, A., K. Hu and D. Riesner. 2001. Transcription of potato spindle tuber viroid by RNA polymerase II starts predominantly at two specific sites. Nucleic acids research, 29: 4589-4597.

Gozmanova, M. 2003. Characterization of the RNA motif responsible for the specific interaction of potato spindle tuber viroid RNA (PSTVd) and the tomato protein Virp1. Nucleic Acids Research, 31: 55345543.

Guner, Ü., H. M. Sipahioglu and M. Usta. 2012. Incidence and genetic stability of Potato spindle tuber pospiviroid in potato in Turkey. Turkish Journal of Agriculture and Forestry, 36: 353-363.

Hammond, R. W. 1992. Analysis of the virulence modulating region of potato spindle tuber viroid (PSTVd) by site-directed mutagenesis. Virology, 187: 654-662.

Hammond, R. W. and R. A. Owens. 1987. Mutational analysis of potato spindle tuber viroid reveals complex relationships between structure and infectivity. Proceedings of the National Academy of Sciences, 84: 3967-3971.

Kasai, A., T. Sano and T. Harada. 2013. Scion on a Stock Producing siRNAs of Potato Spindle Tuber Viroid (PSTVd) Attenuates Accumulation of the Viroid. PLoS ONE, 8: e57736.

Kasschau, K. D., Z. Xie, E. Allen, C. Llave, E. J. Chapman, K. 
A. Krizan and J. C. Carrington. 2003. P1/HC-Pro, a Viral Suppressor of RNA Silencing, Interferes with Arabidopsis Development and miRNA Function. Developmental Cell, 4: 205-217.

Kolonko, N., O. Bannach, K. Aschermann, K.-H. Hu, M. Moors, M. Schmitz, G. Steger and D. Riesner. 2006. Transcription of potato spindle tuber viroid by RNA polymerase II starts in the left terminal loop. Virology, 347: 392-404.

Ling, K. S., R. Li, D. Groth-Helms and F. M. Assis-Filho. 2014. First Report of Potato spindle tuber viroid Naturally Infecting Field Tomatoes in the Dominican Republic. Plant Disease, 98: 701-701. Mackie, A., B. Coutts, M. Barbetti, B. Rodoni, S. McKirdy and R. Jones. 2015. Potato spindle tuber viroid: stability on common surfaces and inactivation with disinfectants. Plant disease, 99: 770-775.

Mahfouze , S. A. 2008. Nucleotide sequence and secondary structure of potato spindle tuber viroid Egyptian strain [electronic resource). Journal of genetic engineering and biotechnology, 6 (1): 21-27.

Owens, R. A. 2007. Potato spindle tuber viroid: the simplicity paradox resolved? Molecular Plant Pathology, 8: 549-560.

Owens, R. A. and T. Baumstark. 2007. Structural differences within the loop E motif imply alternative mechanisms of viroid processing. RNA, 13: 824-834. 